\title{
Bilateral Lung Transplantation Due to Sjögren's Syndrome in a Patient with Klinefelter Syndrome
}

\author{
Beauperthuy Levy Thais, $M D^{1^{*}}$ (D) Anguera de Francisco Gabriel, $M D^{1,2}$, Fonfría Esparcia Carles, $M D^{3}$ \\ and Mancheño Franch $\mathrm{N}, \mathrm{MD}^{4}$
}

\author{
${ }^{1}$ Respiratory Medicine Department, Hospital Universitari I Politècnic La Fe, Spain \\ ${ }^{2}$ Lung Transplant Unit, Hospital Universitari I Politècnic La Fe, Spain \\ ${ }^{3}$ Radiologic Department, Hospital Universitari I Politècnic La Fe, Spain \\ ${ }^{4}$ Pathology Department, Hospital Universitari I Politècnic La Fe, Spain
}

*Corresponding author: Thais Carolina Beauperthuy Levy, Respiratory Medicine Deparment, Hospital Universitari I Politècnic La Fe, Valencia, 46017, Spain

\begin{abstract}
Klinefelter syndrome is one of the most frequent congenital chromosome disorders. Autoimmune diseases are not uncommon in patients with Klinefelter syndrome and associations between them have long been described. However, there is a paucity of literature regarding the effect of hypogonadism on respiratory system in these patients. Herein, we described a 28 -year-old patient who presented with a constellation of unexplained respiratory signs and symptoms, such as tachypnea, hypoxemia, crackles recurrent pulmonary infections since childhood and progressive worsening of his pulmonary function, was diagnosed with Klinefelter syndrome in adulthood. CT chest with diffuse interstitial lung involvement and multiple cystic areas. Based on the radiological findings, an underlying autoimmune process was suspected and investigations showed findings compatible with Sjögren's syndrome. Double lung transplantation was thus indicated considering the irreversible interstitial changes in both lungs. Explant biopsy showed up histological pattern suggestive of interstitial lung disease with autoimmune features. Therefore, the importance of suspecting connective tissue diseases in patients with Klinefelter syndrome, perhaps due to the double $X$ chromosome and a low androgen-toestrogen ratio that characterizes them.
\end{abstract}

\section{Abbrevations}

KS: Klinefelter Syndrome; HRCT: High Resolution Chest Tomography; ANA: Antinuclear Antibodies; Anti-Ds DNA: Anti-Double Stranded DNA; ANCA: Neutrophil Antiplasm Antibodies; HIV: Human Immunodeficiency Virus; AntiSSA/Ro: Autoantibodies Directed Against the Ro/SSA; Anti-SSB: Anti-Sjögren's-Syndrome-Related Antigen B; IPAF: Interstitial Pneumoniae with Autoimmune Features

\section{Introduction}

Klinefelter's syndrome is the most frequent chromosome disorder in males. The genetic background for the KS phenotype is based on the presence of the extra X-chromosome. The affected individual has 47 $\mathrm{XXY}$ chromosomes (classical form) or $46 \mathrm{XY} / 46 \mathrm{XXX}$ (mosaic). The clinical presentation of XXY males may appear in many cases to be similar to that of XY males, and thus it is difficult to make a diagnosis of KS without karyotyping. The rate of diagnosis during childhood is extremely low, and only $10 \%$ of cases are identified before puberty while $25 \%$ of the cases are identified during adulthood [1,2].

Researchers speculate the contributing factor for the observed sexual dimorphims in the pathogenesis of autoimmune diseases [3]. Associations between KS, Systemic Lupus erythematosus, progressive systemic sclerosis, rheumatoid arthritis, Sjögrens's syndrome or mixed connective tissue diseases has long been described, suggesting a possible relationship between female sexual chromosomy, androgen deficiency and autoimmunity $[2,3]$. However, there has no systematic evidence or rationale to account for this fact.

Pulmonary involvement in Klinefelter syndrome is uncommon. Predisposition to infections, an increased risk of pulmonary thromboembolism and a possible association with asthma have been described. 
However, there is a paucity of literature regarding diffuse interstitial lung diseases.

Although many cases of KS associated with autoimmune disease have been described, there are no reports of cases with overlapping connective tissue disease accompanied by severe interstitial lung disease.

\section{Case Report}

A 28-year-old man presented in 2013 with fever, odynophagia, rhinorrhea, cough, sputum's, shortness of breath and hypoxemia. His medical history included an interstitial lung disease from unknown cause since childhood. He also was non-smoker and had a history of osteoporosis, cryptorchidism, gastroesophageal reflux disease and learning disorder.

Physical examination revealed acropachies, gynecomastia and small testes and penis. Moreover, the patient had tall stature with long legs and short trunk. Pulmonary auscultation with bilateral and no edema in lower limbs. Laboratory testing showed low testosterone levels with high levels of luteinizing hormone and follicle-stimulating hormone. A karyotype was performed and chromosomal analysis showed 47, XXY. Thus, he was diagnosed as having Klinefelter syndrome (KS).

X-ray showed a bilateral interstitial pattern. High Resolution Chest Tomography (HRCT) showed a diffuse reticular thickening of the intralobular interstitium with multiple micro and macro thin-walled cysts involving all the lobes (Figure 1). The evolution showed a rapid progression of size and number of cysts and fibrosis. Thus, based on radiological findings and due to the association of autoimmune diseases with Klinefelter syndrome, immunological parameters were performed.

Serologic testing revealed negative Rheumatoid Factor and negative Anti-DsDNA, Antinuclear antibodies (ANA) positive to title 1/80, ANA Spotted Nuclear Pattern, Fine Spotted Cytoplasmic (1/320), Neutrophil Antiplasm Antibodies (ANCA) positive at 1/1280 with atypical cytoplasmic pattern. Serum complement was decreased. Serological test for HIV was negative. Serum immunoglobulins were polyclonal increased and IgG 4 subtype was normal. Positive direct coombs test. High level of cryoglobulin was detected.

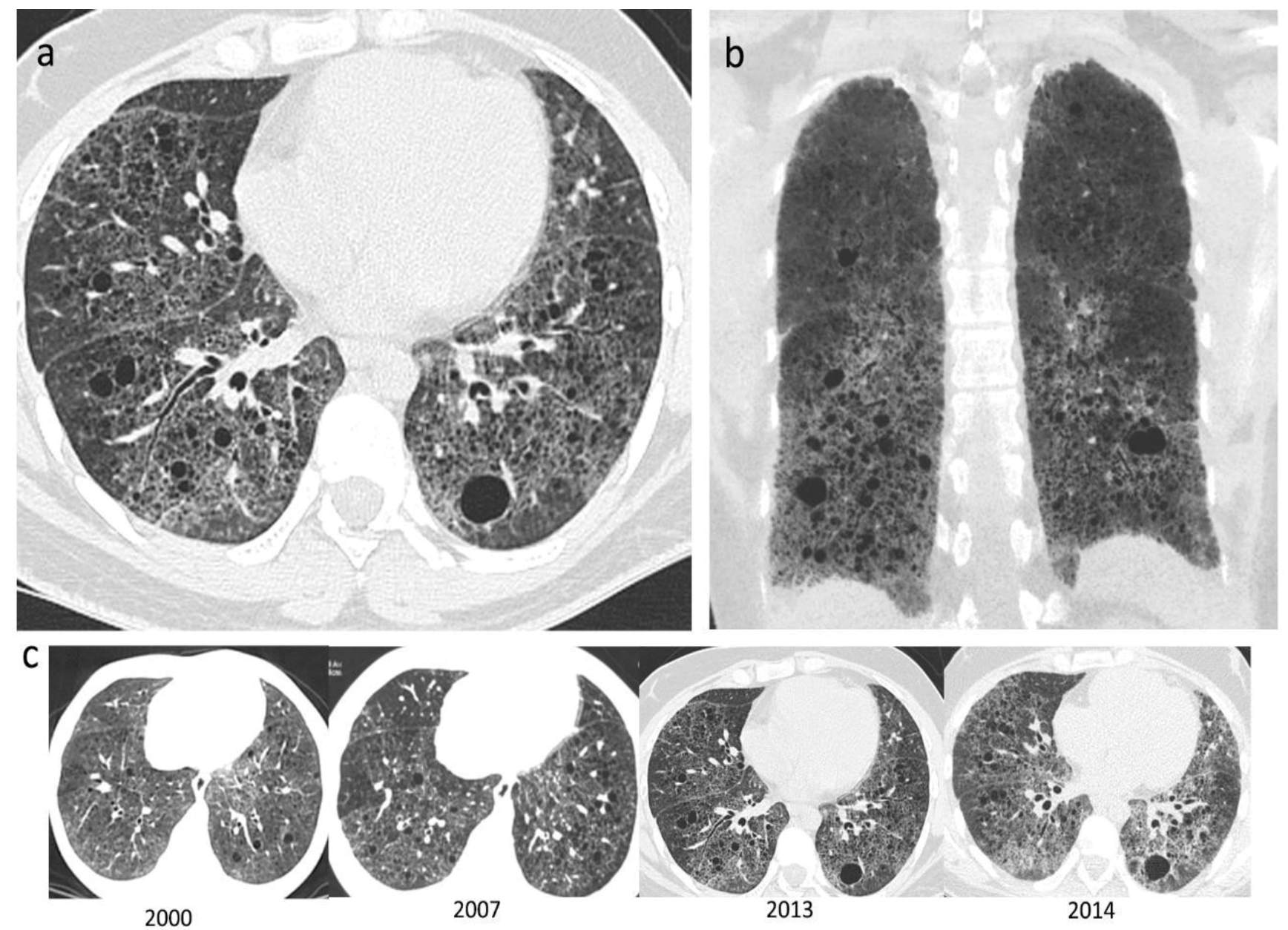

Figure 1: HRCT findings. All images were reconstructed in lung kernel with lung window settings (Width/Level 1500/-600 Hounsfield Units) (a) Axial CT scan at basal level with numerous small and large thin-walled cysts, and reticular opacities representing the thickening of the intralobulbar interstitium; (b) Coronal minimal-intensity projection shows mid to lower lobe predominance; (c) Changes of cysts and reticular opacities of HRCT between baseline (June 2000) and the last study before lung transplantation (August 2014). 
Anti-SSA/Ro was positive but Anti-SSB was negative. The patient reported chronic symptoms of troublesome dry eyes and sensation of sand in the eyes. Therefore, in order to identify the underlying disease a Schrimer test and salivary gland scan was performed, resulting in no secretion of tears and reduction in saliva secretion, respectively. Thus, diagnosis of Sjögren's syndrome was made [4].

Pulmonary Function Test included spirometry, diffusing capacity and 6-min Walk Test, demonstrated severe restrictive alteration and decreased diffusion capacity for carbon monoxide. The clinical severity of the patient did not allow invasive studies to obtain a histological diagnosis. The patient was treated with immunosuppressive therapies, immunomodulatory and anti-inflammatory medications, as azathioprine and corticosteroids. However, his respiratory function deteriorated even more. After evaluation by the multidisciplinary lung transplant team, double lung transplantation was performed in July 2016, thus indicated considering the irreversible interstitial changes in both lungs.

Analysis of explanted lungs showed up at microscopic level fibrosis in subpleural and paraseptal distribution alternating with some areas of normal lung and heterogeneous fibrosis with dense scarring and honeycombing with collapse of alveolar walls and formation of cystic spaces; fibroblastic foci at the edges of dense scars, smooth muscle proliferation and type 2 pneumocyte hyperplasia. Several lymphoid aggregates with germinal centers located within visceral pleura and central airways (Figure 2A, Figure 2B and Figure 2C).

Some areas showed capillaritis with diffuse alveolar hemorrhage (Figure 2B), hemosiderin laden macrophages and giant cells with cholesterol clefts (Figure 2C). Presence of vascular encrustation by hemosiderin (Figure 2D). The arteries show medial hypertrophy and occasional concentric cellular proliferation of intima.
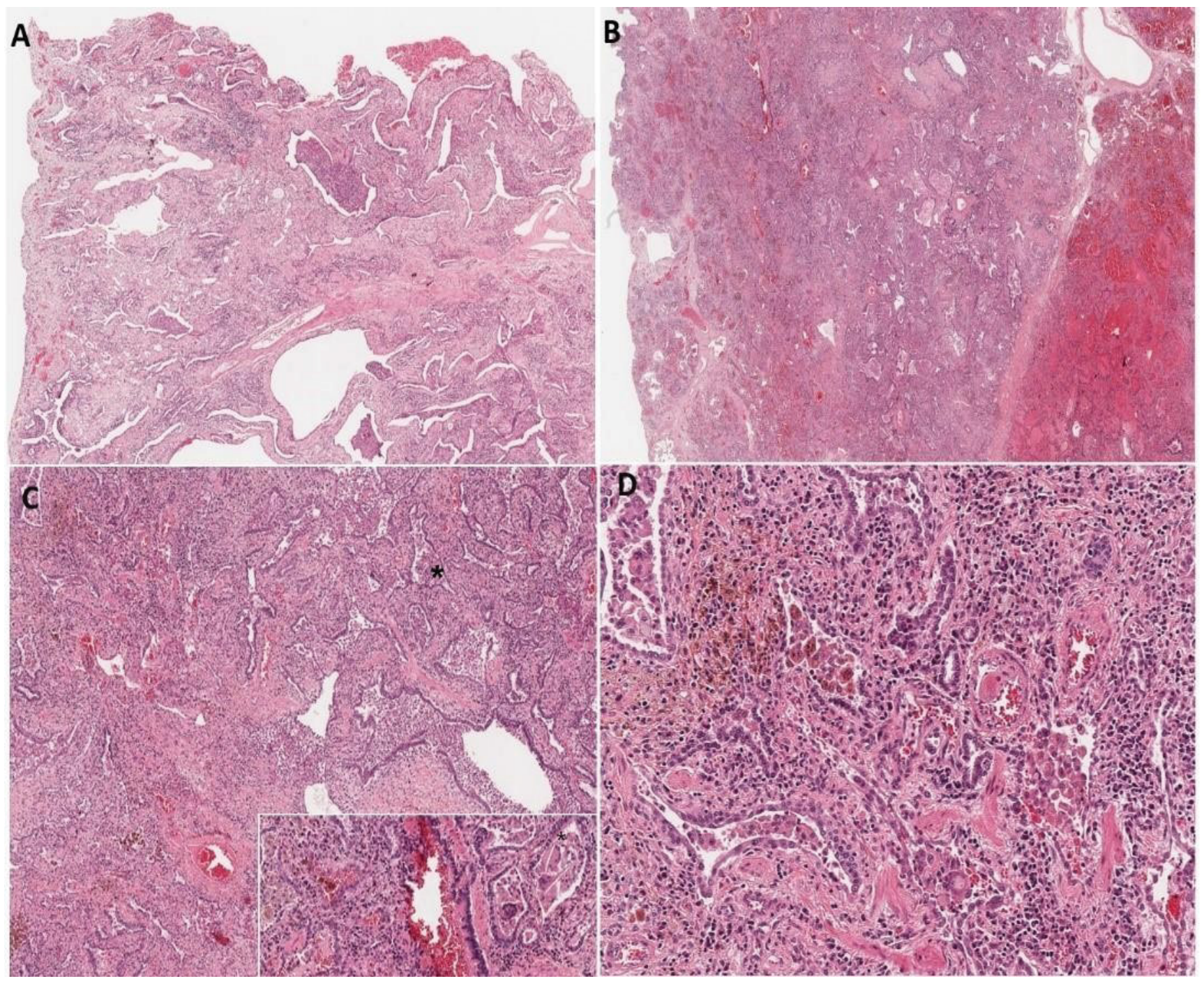

Figure 2: Photomicrograph showing associated key findings (a) Temporally heterogeneous fibrosis with dense scarring and honeycombing with collapse of alveolar walls and formation of cystic spaces; there are fibroblastic foci at the edges of dense scars, smooth muscle proliferation and type 2 pneumocyte hyperplasia. H-E 2X; (b) Capillaritis with diffuse alveolar hemorrhage, hemosiderin laden macrophages and giant cells. H-E 4X; (c) Giant cells with cholesterol clefts ( $\left.{ }^{*}\right) . H-E$ 10X; (d) Hemosiderin laden macrophages and giant cells. H-E 40X. 
One year after lung transplantation he had a diffuse alveolar damage of unknown cause. Thus, his pulmonary function was impaired but 5 years after lung transplantation the patient is alive and his autoimmune disease has not affected other organs, the only symptoms attributable to this involvement have been arthromyalgia without arthritis.

\section{Discussion}

Although numerous theories have been suggested, there has yet not been a clear understanding as to why women overrepresented in almost all autoimmune diseases. It is a general perception that oestrogen may play a role in the pathogenesis of many autoimmune diseases, whereas androgens reduce autoimmune sequelae. Klinefelter syndrome (KS) is the most frequent chromosome disorder in males. It is difficult to make a diagnosis of KS without karyotyping. The rate of diagnosis during childhood is extremely low, and only $10 \%$ of cases are identified before puberty while $25 \%$ of the cases are identified during adulthood [1,2].

Little is known about the immunological features of patients with Klinefelter syndrome. Researchers speculate the contributing factor for the observed sexual dimorphims in the pathogenesis of autoimmune diseases [3]. There is a gene dose effect for the $X$ chromosome such that $46, X X$ women and $47, X X Y$ men share a similar risk of Systemic Lupus erythematosus [2]. Associations between KS with inflammatory rheumatic diseases as progressive systemic sclerosis, rheumatoid arthritis, juvenile idiopathic arthritis, psoriatic arthritis, polymyositis/dermatomyositis, systemic lupus erythematosus, systemic sclerosis, antiphospholipid syndrome, ankylosing spondylitis, Sjögren's syndrome and mixed connective tissue disease [3] has long been described, suggesting a possible relationship between female sexual chromosomy, androgen deficiency and autoimmunity $[2,3]$. However, there has no systematic evidence or rationale to account for this fact. Although many cases of KS associated with autoimmune disease have been described, there are no reports of cases with overlapping connective tissue disease accompanied by severe interstitial lung disease.

It has been proposed that men with Klinefelter's syndrome $(47, \mathrm{XXY})$ are disproportionately higher (1:34) in primary Sjögren's syndrome compared to healthy controls (1:1254) and what is observed in the population (1:500) [4]. Elevated serum levels of IgG and IgM have been reported in some patients with KS and are associated with the presence of anti-RO [4].

It has been reported an overlapping case of IgG4Related Disease and Klinefelter's Syndrome with LupusLike serological features with no lung's involvement [5].

Despite the paucity of literature regarding the relationship between Klinefelter syndrome and lung diseases, it has been reported one case of a congenital pulmonary airway malformation associated with mosaic Klinefelter syndrome and it was diagnosed during pregnancy due to a fetal malformation [6]. Another case of Klinefelter syndrome and pulmonary involvement was reported in a young patient with bronchial asthma and frequent exacerbations whose clinical course was improved after androgen replacement therapy, suggesting that (Th2) cell-driven inflammatory disease, can occur in KS probably due to the negative impact of hypogonadism on lung function [7].

To the best of our knowledge, only one single case of $47, X X Y$ men associated with mixed connective tissue disease and bilateral multiple cystic lesions with lung fibrosis has been reported [8].

On the other hand, forms of diffuse lung disease in older children including the genetic causes of immune deregulation like ANCA-associated vasculitis, are gaining momentum. Differential diagnoses involve neuroendocrine cell hyperplasia of infancy, disorders of surfactant metabolism [9]. In this case, due to the absence of family illnesses and the course of disease, the genetic study was not carried out.

The clinical severity of the patient did not allow invasive studies to obtain a histological diagnosis. Based on the clinical symptoms of xerostomia and xerophthalmia, along with serologic detection of antinuclear antibodies, anti- SSA/RO, radiographic findings and the explant histopathology, we submit that these findings can be sufficiently distinctive to obviate the need for lip biopsy in order to confirm a Sjögren's syndrome, therefore, an interstitial lung disease in Sjögren's syndrome was suggested [10].

Furthermore, another differential diagnosis is IgG4related which often presents as a multiorgan disease and may be confused with malignancy, infection or other immune-mediated conditions ANCAs). Our patient had normal serum levels of IgG4. Moreover, positive SSA/Ro which is considered as an exclusion criterion of the disease according to the 2019 American College of Rheumatology/European League against Rheumatism classification criteria for IgG4-related disease.

Diffuse cystic lung disease is an uncommon clinical and radiographic presentation with a broad differential diagnosis. Obtaining a thorough history and performing a physical examination are essential for establishing DCLD diagnosis. Extrapulmonary findings, especially in the skin and kidneys, provide important diagnostic clues. In our case, due to the radiological findings with atypical fibrosis at an early age and multiple cystic lung involvement, as they were not characteristic of any particular pathology, an attempt was made to identify the underlying cause. The main diagnosis was Lymphoid Interstitial Pneumonia. This is one of the most common lymphocyte predominant pulmonary pathologies associated with interstitial pneumonia with 
autoimmune features. Its presence should raise the suspicion for an underlying autoimmune process [11].

There has been an emerging interest in the role of lung transplantation in the management of interstitial lung disease secondary to connective tissue diseases. It is important to note that it is rare to perform lung transplantation for this indication. After 5 years of transplantation, the patient is still alive, without relevant complications related to his autoimmune disease. Therefore, lung transplantation seems a valid treatment option in a case with similar characteristics.

\section{Conclusion}

Hormonal conditions similar to female conditions are likely to precipitate the development of an autoimmune disease or worsen its progression as happened in this patient. Interstitial lung diseases can occur in KS probable to an underlying autoimmune process due to the negative impact of hypogonadism. The diagnosis of KS has been delayed in the present case for nearly 30 years. The syndrome remains highly underdiagnosed because of substantial variation in its clinical presentation. Nonetheless, pulmonary physicians should be aware of $\mathrm{KS}$ and the associated immunological features and pulmonary involvement.

\section{References}

1. Shiraishi K, Matsuyama H (2019) Klinefelter syndrome: From pediatrics to geriatrics. Reprod Med Biol 18: 140-150.

2. Sawalha AH, Harley JB, Scofield RH (2009) Autoimmunity and klinefelter's syndrome: When men have two $X$ chromosomes. J Autoimmun 33: 31-34.
3. Rovenský J, Imrich R, Lazúrová I, Payer J (2010) Rheumatic diseases and klinefelter's syndrome. Ann N Y Acad Sci 1193: 1-9.

4. Harris VM, Sharma R, Cavett J, Kurien BT, Liu K, et al. (2016) Klinefelter's syndrome $(47, X X Y)$ is in excess among men with Sjögren's syndrome. Clin Immunol 168: 25-29.

5. Takanashi S, Hanaoka H, Ota Y, Kaneko Y, Takeuchi $T$ (2020) An overlapping case of igg4-related disease and klinefelter syndrome with lupus-like serological and neurological features: A case report and literature review. Intern Med 59: 2601-2609.

6. Holubyeva A, Bracero LA, Feuerstein JL, Bush S (2020) Congenital pulmonary airway malformation associated with mosaic klinefelter syndrome. J Clin Ultrasound 48: 121-124.

7. Ladias S, Katsenos S (2018) Klinefelter syndrome and bronchial asthma: Is there any relationship between the low testosterone levels and asthma exacerbations? Lung India 35: 368-369.

8. Ishihara K, Yoshimura M, Nakao H, Kanakura Y, Kanayama $Y$, et al. (1994) $T$ cell abnormalities in mixed connective tissue disease complicated with klinefelter's syndrome. Intern Med 33: 714-717.

9. Vece TJ, Young LR (2016) Update on diffuse lung disease in children. Chest 149: 836-845.

10. Shiboski $\mathrm{CH}$, Shiboski SC, Seror R, Criswell LA, Labetoulle $\mathrm{M}$, et al. (2017) 2016 American college of rheumatology/ european league against rheumatism classification criteria for primary sjögren's syndrome: A consensus and datadriven methodology involving three international patient cohorts. Ann Rheum Dis 76: 9-16.

11. Fischer A, Antoniou KM, Brown KK, Cadranel J, Corte TJ, et al. (2015) An official european respiratory society/american thoracic society research statement: Interstitial pneumonia with autoimmune features. Eur Respir J 46: 976-987. 